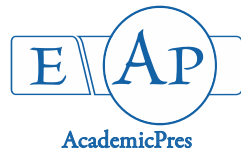

\title{
Evaluation of In Vitro Shoot Elongation and Rooting of Date Palm, and Determination of Physiological Characteristics of Regenerated Plantlets
}

\author{
Reda MEZIANI ${ }^{1 *}$, Mouaad Amine MAZRI ${ }^{2}$, Mahassine ARHAZZAL ${ }^{1,3}$, \\ Ilham BELKOURA ${ }^{3}$, Chakib ALEM ${ }^{4}$, Fatima JAITI ${ }^{4}$ \\ ${ }^{I}$ Institut National de la Recherche Agronomique, CRRA-Errachidia, UR Systemes Oasiens, Laboratoire National de Culture des Tissus du \\ PalmierDattier, BP 2, Errachidia, Morocco; redameziani@yahoo.fr ("corresponding author) \\ ${ }^{2}$ Institut National de la Recherche Agronomique, CRRA-Marrakech, UR Agro-Biotechnologie, Laboratoire de Biotechnologie Végétale, BP 533, \\ Marrakech,Morocco;m.a.mazri@gmail.com \\ ${ }^{3} E c o l e$ Nationale d'Agriculture, Département des Sciences de Base, Laboratoire de Culture In Vitro, BP S/40, Meknes, \\ Morocco;mahassine.ar@gmail.com; bilham@enameknes.ac.ma \\ ${ }^{4}$ Université Moulay Ismail, Faculté des Sciences et Techniques, BP 509, Errachidia, Morocco; alem04@yahoo.fr; fatimajaiti@yahoo.fr
}

\begin{abstract}
The effects of various culture conditions on shoot elongation, rooting and plantlet acclimatization were tested. Adventitious shoots obtained through direct organogenesis of date palm (Phoenix dactylifera L.) cv. 'Mejhoul' were used as explants. The effects of culture medium texture, plant growth regulators, polyvinylpyrrolidone, adenine, myo-inositol, Lglutamine, and carbon source on in vitro plantlet quality and subsequent acclimatization were evaluated. The most effective culture medium was the semi-solid and half-strength Murashige and Skoog medium without plant growth regulators, supplemented with $30 \mathrm{~g} \mathrm{~L}^{-1}$ sucrose. After 3 months of culture on this medium, the average shoot length was $13.6 \mathrm{~cm}$, the average number of adventitious roots per shoot was 3.6, and the average root length was $3.85 \mathrm{~cm}$. The survival rate of these plantlets in acclimatization was $90 \%$. On the other hand, liquid medium, plant growth regulators, polyvinylpyrrolidone, adenine, myo-inositol and L-glutamine did not increase the survival rate during acclimatization. Along with these experiments, some physiological characteristics of the plantlets obtained in vitro were also determined. Chlorophyll content and fluorescence, foliar surface and stomatal conductance were measured after 3 months of culture in each medium. The ranges were as follows: Chlorophyll content, 11.7-31.8 CCI; chlorophyll fluorescence, 0.633-0.795; foliar surface, $7.35-13.29 \mathrm{~cm}^{2}$; and stomatal conductance, $10.3-36.0 \mathrm{mmol} \mathrm{m}^{-2} \mathrm{~s}^{-1}$. Interestingly, positive correlations between the physiological characteristics of the plantlets and their survival percentage in the glasshouse were revealed. The findings of this investigation will be valuable for large-scale and cost-saving production of date palm cv. 'Mejhoul' plants.
\end{abstract}

Keywords: chlorophyll content; chlorophyll fluorescence; foliar surface; morphological characteristics; organogenesis; Phoenix dactylifera L.; stomatal conductance

Abbreviations: 2iP, 6-(dimethylallylamino) purine; BAP, 6-benzylaminopurine; CCI, chlorophyll content index; CGS, commercial granulated sugar; $\mathrm{GA}_{3}$, gibberellic acid; IAA, indole-3-acetic acid; IBA, indole-3-butyric acid; MS, Murashige and Skoog; NAA, 1-naphthaleneacetic acid; NOA, 2-naphthoxyacetic acid; PGR, plant growth regulator; PVP, polyvinylpyrrolidone.

\section{Introduction}

Date palm (Phoenix dactylifera L.) belongs to the genus Phoenix, which consists of 14 species (Johnson, 2011). It is mainly grown in the MENA region but also distributed in other countries such as Spain, Australia and the USA (Shabani et al., 2014). Date palm is one of the most commercially important fruit species in the Middle East and
North Africa. It is cultivated for its edible fruits and is known to play roles in creating equable microclimates within oasis ecosystems and generating employment (Jain, 2012). In Morocco, date palm is mainly grown in preSaharan arid regions with about 453 cultivars (Sedra, 2015).

'Mejhoul' cv. (also known as 'Medjool') is one of the best and the most sought after date palm varieties in the world (Sedra, 2015). In Morocco, cv. 'Mejhoul' is threatened by 
78

Bayoud, a vascular disease caused by the pathogen Fusarium oxysporum f. sp. albedinis. Unfortunately, Bayoud has significantly decreased the population of cv. 'Mejhoul' (Sedra, 2011). Nowadays, the only effective way to preserve this variety is by rapid and large-scale propagation through in vitro culture techniques, followed by plantation in Bayoud-free areas.

Date palm can be micropropagated through either somatic embryogenesis or organogenesis (Mazri and Meziani, 2015). In Morocco, organogenesis is the mainly used technique since it allows production of true-to-type plants (Sedra, 2005; Meziani et al., 2015). Over the last years, organogenesis has played a major role in the propagation of date palm genotypes of commercial interest.

The organogenesis pathway involves four steps: adventitious bud initiation, shoot bud multiplication, shoot elongation and rooting, and plantlet acclimatization (Mazri and Meziani, 2013). In previous papers, the effects of different culture conditions on adventitious bud initiation (Meziani et al., 2016) and shoot bud multiplication of date palm cv. 'Mejhoul' (Meziani et al., 2015; 2016; Mazri et al., 2016) were reported. Studies on in vitro shoot elongation and rooting of date palm are scarce. Although there are some studies dealing with the effects of medium composition and growth conditions on in vitro shoot elongation and rooting in some date palm cultivars (Khierallah and Bader, 2007; Khan and Bi Bi, 2012; Mazri, 2013; Mazri and Meziani, 2013), it is well known that in vitro propagation of date palm depends on the genotype (Zaid et al., 2011).

Chlorophyll content, foliar surface and stomatal conductance are physiological characteristics that can affect plantlet quality, survival, growth and development after transplantation. Chlorophyll is a photoreceptor, an indicator of the photosynthetic potential of plants and a catalyst for the conversion of sunlight into chemical energy. It plays a major role in the photochemical synthesis of carbohydrates (Vernon and Seely, 1966; Oliveira et al., 2016). Plant foliar surface is a physiological index for evaluating some metabolic processes such as growth, transpiration, photosynthesis and respiration. It also constitutes a boundary between plant tissues and the environment and plays a major role in the defense against pathogen infections (Derridj et al., 1996; Lee et al., 2008; Camen et al., 2010). Stomatal conductance is an important factor in controlling leaf transpiration, regulating water losses by the plant and in the emission and uptake of acids (Burrows and Milthorpe, 1976; Cabrera et al., 1995; Filella et al., 2009). It is also an indicator of stomatal opening and photosynthesis activity (Jones, 1992; Johnson et al., 1979).

Herein, the effects of different groups of factors on shoot elongation and rooting, and plantlet acclimatization of date palm cv. 'Mejhoul' were investigated. Accordingly, the effects of medium texture, plant growth regulators (PGRs), carbon sources, polyvinylpyrrolidone (PVP), Lglutamine, myo-inositol and adenine were tested. Furthermore, the chlorophyll content and fluorescence, foliar surface and stomatal conductance of the in vitro plants were measured and their correlations with acclimatization were determined.

\section{Materials and Methods}

\section{Origin of plant material}

In vitro young plantlets of date palm cv. 'Mejhoul' were obtained according to Meziani et al. (2015; 2016). Briefly, shoot tips of date palm (Phoenix dactylifera L.) cv. 'Mejhoul' were obtained from 3-year-old offshoots collected from a date palm orchard in Erfoud, Morocco. The shoot tips were disinfected and explants from meristematic tissues were extracted as described by Mazri et al. (2016). The explants were cultured on half-strength Murashige and Skoog (1962) medium (MS/2, consisting of $1 / 2$ MS macro-elements, MS microelements and MS vitamins) supplemented with 14.2 $\mu \mathrm{M}$ indole-3-acetic acid (IAA), $13.4 \mu \mathrm{M} \quad 1$ naphthaleneacetic acid (NAA), $0.5 \mu \mathrm{M} \quad 6-$ (dimethylallylamino) purine (2iP), $1.5 \mathrm{~g} \mathrm{~L}^{-1} \mathrm{PVP}$ (Sigma, Steinheim, Germany), $30 \mathrm{~g} \mathrm{~L}^{-1}$ sucrose (Sigma, St. Louis, MO, USA) and $6 \mathrm{~g} \mathrm{~L}^{-1}$ agar (Sigma, St. Louis, MO, USA) for 9 months under dark conditions at $25 \pm 1{ }^{\circ} \mathrm{C}$, with monthly subcultures. The formed shoot buds were cultured for 3 months (with 1-month subculture intervals) on MS/2 medium supplemented with $0.9 \mu \mathrm{M}$ 2-naphthoxyacetic acid (NOA), $1.1 \mu \mathrm{M}$ IAA, $1.8 \mu \mathrm{M}$ kinetin, $1.9 \mu \mathrm{M} 2 \mathrm{iP}, 1 \mathrm{~g} \mathrm{~L}^{-1}$ PVP, $30 \mathrm{~g} \mathrm{~L}^{-1}$ commercial granulated sugar (CGS; Cosumar, Casablanca, Morocco), and $6 \mathrm{~g} \mathrm{~L}^{-1}$ agar, under a $16 \mathrm{~h}$ light photoperiod (13.5 $\mu \mathrm{mol} \mathrm{m}^{-2} \mathrm{~s}^{-1}$ light intensity). Afterwards, young plantlets of about $4-5 \mathrm{~cm}$ length and one root were singled out and used for the subsequent experiments.

\section{Effects of medium components on shoot elongation and rooting}

In the first experiment, the effects of medium texture on shoot elongation and rooting were evaluated. Shoots were cultured on MS/2 medium supplemented with $30 \mathrm{~g} \mathrm{~L}^{-1}$ CGS. The medium was either semi-solid (CM1) or liquid (CM2).

In the second experiment, the effects of various PGR combinations were tested (Table 1). Based on the results from the first experiment, indole-3-butyric acid (IBA), NAA, 6-benzylaminopurine (BAP) and kinetin were added to semi-solid MS/2 medium either alone or in combination at the concentration of $1 \mathrm{mg} \mathrm{L}^{-1}$ each, while filter-sterilized gibberellic acid $\left(\mathrm{GA}_{3}\right)$ was added to the medium at $1 \mathrm{mg} \mathrm{L}^{-1}$ after autoclaving. All culture media used in this experiment were supplemented with $30 \mathrm{~g} \mathrm{~L}^{-1}$ CGS.

In the third experiment, the effects of PVP, adenine, myo-inositol and L-glutamine were evaluated at the concentrations of $1 \mathrm{~g} \mathrm{~L}^{-1}$ each (Table 1); again, semi-solid $\mathrm{MS} / 2$ medium supplemented with $30 \mathrm{~g} \mathrm{~L}^{-1} \mathrm{CGS}$ was used.

Finally, the effects of various carbon sources on shoot elongation and rooting were evaluated. Semi-solid MS/2 medium was supplemented with sucrose, sorbitol or mannitol at $30 \mathrm{~g} \mathrm{~L}^{-1}$ (all purchased from Sigma, St. Louis, MO, USA; Table 1).

\section{Culture conditions}

In all experiments, the culture medium used was MS/2. When semi-solid medium was required, $6 \mathrm{~g} \mathrm{~L}^{-1}$ agar was added. The $\mathrm{pH}$ of all media was adjusted to 5.8 and $40 \mathrm{ml}$ volumes were dispensed into jars $(12 \mathrm{~cm}$ in height and 6.5 $\mathrm{cm}$ in diameter) before autoclaving at $121{ }^{\circ} \mathrm{C}$ for $25 \mathrm{~min}$. 
The cultures were kept at $25^{\circ} \mathrm{C}$ under $16 \mathrm{~h}$ photoperiod $\left(40 \mu \mathrm{mol} \mathrm{m} \mathrm{m}^{-2} \mathrm{~s}^{-1}\right)$. In all experiments, shoots were cultured for 3 months with transfer to fresh medium at 1-month intervals.

\section{Physiological characteristics determination Chlorophyll content determination}

The chlorophyll content was measured after 3 months of culture on elongation-rooting media using a CCM-200 Chlorophyll Content Meter (Opti-Sciences, Tyngsboro, Massachusetts, USA). The measurements were performed on fully expanded leaves (one leaf per plantlet and 10 plantlets per treatment). The chlorophyll content was expressed as chlorophyll content index (CCI), which is the ratio of percent transmission at $931 \mathrm{~nm}$ to $653 \mathrm{~nm}$ through a leaf sample.

\section{Chlorophyll fluorescence determination}

The chlorophyll fluorescence, representing the photochemical capacity of Photosystem II, was measured after 3 months of culture on elongation-rooting media using a chlorophyll fluorometer (OS-30p, Opti- Science Inc., Hudson, NH, USA). Briefly, the plant leaves were dark adapted for $30 \mathrm{~min}\left(25^{\circ} \mathrm{C}\right)$ then initial fluorescence (Fo), maximum fluorescence $(\mathrm{Fm})$ and their ratio $(\mathrm{Fv} / \mathrm{Fm}$; where $\mathrm{Fv}$ is the difference between Fm and Fo) were calculated. For each treatment, one leaf per plantlet and 10 plantlets were used.

\section{Foliar surface determination}

The foliar surface of one leaf per plantlet and 10 plantlets per treatment was measured using a digital planimeter (Ushikata X-PLAN 380 dIII, Japan) after 3 months of culture on elongation-rooting media.

\section{Stomatal conductance measurement}

Stomatal conductance was measured with an AP4 porometer (Delta-T Devices, Cambridge, UK) after 3 months of culture on elongation-rooting media, using one leaf per plantlet and 10 plantlets per treatment.

\section{Plantlet acclimatization}

The obtained plantlets were acclimatized as described by Mazri et al. (2017). Briefly, the plantlets were removed from the culture media and the root system was washed with tap water. The roots were immersed for $15 \mathrm{~min}$ in a solution of $1 \mathrm{~g} \mathrm{~L}^{-1}$ Pelt 44 PM systemic fungicide (Bayer Crop Science, Bayer Maghreb SA, Casablanca, Morocco) then the plantlets were transferred to plastic bags containing a mixture of peat and gravel $(1: 1, \mathrm{w} / \mathrm{w})$. The plantlets were sprayed with a solution of $0.5 \mathrm{~g} \mathrm{~L}^{-1}$ Pelt $44 \mathrm{PM}$ then covered with a polyethylene bag. The polyethylene was gradually removed to allow the plantlets to acclimatize to glasshouse conditions $\left(27^{\circ} \mathrm{C}, 70 \%\right.$ relative humidity).

Table 1. Composition of culture media used in this study

\begin{tabular}{|c|c|c|c|c|c|c|c|c|c|c|c|c|c|c|c|}
\hline $\begin{array}{l}\text { Exper } \\
\text { iment }\end{array}$ & $\begin{array}{l}\text { Culture } \\
\text { medium }\end{array}$ & Agar & IBA & NAA & BAP & KIN & $\mathrm{GA}_{3}$ & CGS & Sucrose & Sorbitol & Mannitol & PVP & Adenine & $\begin{array}{l}\text { Myo- } \\
\text { inositol }\end{array}$ & $\begin{array}{c}\text { L- } \\
\text { Gluta } \\
\text { mine }\end{array}$ \\
\hline \multirow{2}{*}{1} & CMI & + & - & - & - & - & - & + & - & - & - & - & - & - & - \\
\hline & CM2 & - & - & - & - & - & - & + & - & - & - & - & - & - & - \\
\hline \multirow{10}{*}{2} & CM3 & + & + & - & + & - & - & + & - & - & - & - & - & - & - \\
\hline & CM4 & + & + & - & - & + & - & + & - & - & - & - & - & - & - \\
\hline & CM5 & + & - & + & + & - & - & + & - & - & - & - & - & - & - \\
\hline & CM6 & + & - & + & - & + & - & + & - & - & - & - & - & - & - \\
\hline & CM7 & + & + & + & + & - & - & + & - & - & - & - & - & - & - \\
\hline & CM8 & + & + & + & - & + & - & + & - & - & - & - & - & - & - \\
\hline & CM9 & + & + & - & + & + & - & + & - & - & - & - & - & - & - \\
\hline & CM10 & + & - & + & + & + & - & + & - & - & - & - & - & - & - \\
\hline & CM11 & + & + & + & + & + & - & + & - & - & - & - & - & - & - \\
\hline & CM12 & + & - & - & - & - & + & + & - & - & - & - & - & - & - \\
\hline \multirow{15}{*}{3} & CM13 & + & - & - & - & - & - & + & - & - & - & + & + & + & + \\
\hline & CM14 & + & - & - & - & - & - & + & - & - & - & + & + & + & - \\
\hline & CM15 & + & - & - & - & - & - & + & - & - & - & + & + & - & + \\
\hline & CM16 & + & - & - & - & - & - & + & - & - & - & + & + & - & - \\
\hline & CM17 & + & - & - & - & - & - & + & - & - & - & + & - & + & + \\
\hline & CM18 & + & - & - & - & - & - & + & - & - & - & + & - & + & - \\
\hline & CM19 & + & - & - & - & - & - & + & - & - & - & + & - & - & + \\
\hline & CM20 & + & - & - & - & - & - & + & - & - & - & + & - & - & - \\
\hline & CM21 & + & - & - & - & - & - & + & - & - & - & - & + & + & + \\
\hline & $\mathrm{CM} 22$ & + & - & - & - & - & - & + & - & - & - & - & + & + & - \\
\hline & $\mathrm{CM} 23$ & + & - & - & - & - & - & + & - & - & - & - & + & - & + \\
\hline & CM24 & + & - & - & - & - & - & + & - & - & - & - & + & - & - \\
\hline & CM25 & + & - & - & - & - & - & + & - & - & - & - & - & + & + \\
\hline & CM26 & + & - & - & - & - & - & + & - & - & - & - & - & + & - \\
\hline & CM27 & + & - & - & - & - & - & + & - & - & - & - & - & - & + \\
\hline \multirow{3}{*}{4} & CM28 & + & - & - & - & - & - & - & + & - & - & - & - & - & - \\
\hline & CM29 & + & - & - & - & - & - & - & - & + & - & - & - & - & - \\
\hline & CM30 & + & - & - & - & - & - & - & - & - & + & - & - & - & - \\
\hline
\end{tabular}


80

\section{Statistical analysis}

In all experiments, four young plantlets per jar were used. Each jar was considered as one experimental unit and was repeated 10 times. Therefore, 40 young plantlets were used in each medium treatment. The experiments were established as a completely randomized design. After 3 months of culture, the following data were recorded: shoot length, root number and length, chlorophyll content and fluorescence, foliar surface, and stomatal conductance. The data are reported as means \pm standard deviations. Three months after ex vitro transfer, the survival rate of plantlets was calculated.

Data were subjected to analysis of variance. Significant differences between means were assessed with the StudentNewman-Keuls test at the 0.05 significance level using SPSS for windows (SPSS v.21, IBM, Chicago, IL, USA). Percentage data were analyzed after arcsine transformation and correlations between physiological characteristics and plantlet acclimatization were evaluated using Pearson's correlation coefficients.

\section{Results}

Effects of medium texture on shoot elongation, rooting and plantlet acclimatization

After 3 months of culture, there was a significant difference in shoot length between semi-solid (CM1) and liquid (CM2) media, while no significant difference was found in terms of root number and length. The average shoot length was $13.15 \mathrm{~cm}$ in CM1 medium while it was $16.55 \mathrm{~cm}$ in CM2 medium. The average number of roots per shoot was $3.3(3.15 \mathrm{~cm}$ length) in CM1 medium and 3.7 (3.45 cm length) in CM2 medium. Interestingly, the shoots cultured on CM1 medium were thicker, greener and more vigorous than those cultured on CM2 medium. As regards to the physiological characteristics, the statistical analysis revealed significant differences in terms of chlorophyll content, chlorophyll fluorescence, foliar surface and stomatal conductance between CM1 and CM2 medium (Table 2). In addition, three months after transfer to the glasshouse, plantlets derived from shoots cultured on semisolid medium survived at a significantly higher rate (85\%) than those derived from shoots cultured on liquid medium (40\%). The results of this first experiment showed that despite that liquid medium resulted in longer plantlets; those developed on semi-solid medium were physiologically superior and survived well under glasshouse conditions.

Effects of PGRs on shoot elongation, rooting and plantlet acclimatization

Generally speaking, PGRs induced longer plantlets with more roots compared to PGR-free medium (CM1). The highest shoot length $(19.2 \mathrm{~cm})$ was observed on CM6 medium, which was containing NAA and kinetin. The average number of roots ranged from 3.9 (CM12 medium) to 5.9 (CM7 medium) while root length ranged from 5.20 $\mathrm{cm}$ (CM12 medium) to $6.20 \mathrm{~cm}$ (CM9 and CM10 media). There was no significant difference in shoot rooting among all tested media (Table 3). The ranges of physiological characteristics were: chlorophyll content, 11.7-14.4 CCI; chlorophyll fluorescence, 0.685-0.704; foliar surface, 9.01$10.19 \mathrm{~cm}^{2}$; and stomatal conductance, $10.3-15.6 \mathrm{mmol} \mathrm{m}^{-2}$ $\mathrm{s}^{-1}$. The survival rates ranged from 50 to $70 \%$. Compared to the first experiment, the shoots obtained from CM1 medium exhibited a higher survival rate than those cultured on media containing PGRs. Consequently, these findings suggest using a PGR-free medium for shoot elongation and rooting since it permits cost saving as well as high survival after acclimatization to ex vitro conditions.

Table 2. Effect of medium texture on shoot elongation, rooting, physiological characteristics and plantlet acclimatization

\begin{tabular}{|c|c|c|c|c|c|c|c|c|}
\hline $\begin{array}{l}\text { Culture } \\
\text { medium }\end{array}$ & $\begin{array}{c}\text { Shoot length } \\
(\mathrm{cm})\end{array}$ & $\begin{array}{c}\text { Number of } \\
\text { roots per shoot }\end{array}$ & $\begin{array}{l}\text { Root length } \\
(\mathrm{cm})\end{array}$ & $\begin{array}{c}\text { Chlorophyll } \\
\text { content }(\mathrm{CCI})\end{array}$ & $\begin{array}{l}\text { Chlorophyll } \\
\text { fluorescence }\end{array}$ & $\begin{array}{l}\text { Foliar surface } \\
\qquad\left(\mathrm{cm}^{2}\right)\end{array}$ & $\begin{array}{l}\text { Stomatal condu } \\
\text { ctance } \\
\left(\mathrm{mmol} \mathrm{m}^{-2} \mathrm{~s}^{-1}\right)\end{array}$ & $\begin{array}{c}\text { Plantlet } \\
\text { acclimatization }\end{array}$ \\
\hline CM1 & $13.15 \pm 1.36 \mathrm{a}$ & $3.3 \pm 0.82 \mathrm{a}$ & $3.15 \pm 1.23 \mathrm{a}$ & $31.6 \pm 3.00 \mathrm{a}$ & $0.726 \pm 0.034 \mathrm{a}$ & $13.03 \pm 0.69 a$ & $34.6 \pm 7.05 a$ & $85 \pm 36.6 a$ \\
\hline CM2 & $16.55 \pm 2.30 \mathrm{~b}$ & $3.7 \pm 1.49 \mathrm{a}$ & $3.45 \pm 1.04 \mathrm{a}$ & $12.3 \pm 0.97 b$ & $0.633 \pm 0.053 b$ & $7.35 \pm 1.26 b$ & $16.4 \pm 7.48 b$ & $40 \pm 50.3 b$ \\
\hline
\end{tabular}

Table 3. Effect of plant growth regulators on shoot elongation, rooting, physiological characteristics and plantlet acclimatization

\begin{tabular}{ccccccccc}
\hline $\begin{array}{c}\text { Culture } \\
\text { medium }\end{array}$ & $\begin{array}{c}\text { Shoot length } \\
(\mathrm{cm})\end{array}$ & $\begin{array}{c}\text { Number of } \\
\text { roots per shoot }\end{array}$ & $\begin{array}{c}\text { Root length } \\
(\mathrm{cm})\end{array}$ & $\begin{array}{c}\text { Chlorophyll } \\
\text { content }(\mathrm{CCI})\end{array}$ & $\begin{array}{c}\text { Chlorophyll } \\
\text { fluorescence }\end{array}$ & $\begin{array}{c}\text { Foliar surface } \\
\left(\mathrm{cm}^{2}\right)\end{array}$ & $\begin{array}{c}\text { Stomatal condu } \\
\text { ctance } \\
\left(\mathrm{mmol} \mathrm{m}^{-2} \mathrm{~s}^{-1}\right)\end{array}$ & $\begin{array}{c}\text { Plantlet } \\
\text { acclimatization }\end{array}$ \\
\hline $\mathrm{CM} 3$ & $15.60 \pm 2.57 \mathrm{a}$ & $4.4 \pm 1.07 \mathrm{a}$ & $5.30 \pm 1.18 \mathrm{a}$ & $11.7 \pm 0.68 \mathrm{a}$ & $0.694 \pm 0.043 \mathrm{a}$ & $9.93 \pm 1.16 \mathrm{a}$ & $10.3 \pm 1.62 \mathrm{a}$ & $65 \pm 48.9 \mathrm{a}$ \\
$\mathrm{CM} 4$ & $15.10 \pm 2.47 \mathrm{a}$ & $4.5 \pm 1.35 \mathrm{a}$ & $5.70 \pm 1.16 \mathrm{a}$ & $13.1 \pm 1.38 \mathrm{ab}$ & $0.688 \pm 0.033 \mathrm{a}$ & $9.54 \pm 1.59 \mathrm{a}$ & $12.5 \pm 1.15 \mathrm{ab}$ & $60 \pm 50.3 \mathrm{a}$ \\
$\mathrm{CM} 5$ & $15.30 \pm 1.70 \mathrm{a}$ & $5.5 \pm 1.51 \mathrm{a}$ & $5.60 \pm 0.84 \mathrm{a}$ & $12.7 \pm 1.46 \mathrm{ab}$ & $0.701 \pm 0.032 \mathrm{a}$ & $9.01 \pm 2.36 \mathrm{a}$ & $14.5 \pm 2.36 \mathrm{~b}$ & $50 \pm 51.3 \mathrm{a}$ \\
$\mathrm{CM} 6$ & $19.20 \pm 2.72 \mathrm{~b}$ & $5.6 \pm 1.51 \mathrm{a}$ & $5.45 \pm 1.09 \mathrm{a}$ & $12.0 \pm 1.02 \mathrm{a}$ & $0.704 \pm 0.029 \mathrm{a}$ & $9.75 \pm 2.14 \mathrm{a}$ & $12.9 \pm 1.82 \mathrm{ab}$ & $60 \pm 50.3 \mathrm{a}$ \\
$\mathrm{CM} 7$ & $15.90 \pm 2.22 \mathrm{a}$ & $5.9 \pm 2.23 \mathrm{a}$ & $5.80 \pm 1.58 \mathrm{a}$ & $13.1 \pm 0.95 \mathrm{ab}$ & $0.697 \pm 0.013 \mathrm{a}$ & $9.83 \pm 1.84 \mathrm{a}$ & $15.4 \pm 3.10 \mathrm{~b}$ & $65 \pm 48.9 \mathrm{a}$ \\
$\mathrm{CM} 8$ & $15.70 \pm 1.77 \mathrm{a}$ & $5.7 \pm 0.95 \mathrm{a}$ & $5.90 \pm 1.37 \mathrm{a}$ & $12.5 \pm 0.76 \mathrm{ab}$ & $0.699 \pm 0.015 \mathrm{a}$ & $9.45 \pm 2.47 \mathrm{a}$ & $13.7 \pm 1.89 \mathrm{~b}$ & $70 \pm 47.0 \mathrm{a}$ \\
$\mathrm{CM} 9$ & $17.20 \pm 1.93 \mathrm{ab}$ & $4.4 \pm 1.51 \mathrm{a}$ & $6.20 \pm 1.27 \mathrm{a}$ & $12.2 \pm 0.96 \mathrm{a}$ & $0.700 \pm 0.017 \mathrm{a}$ & $9.79 \pm 2.79 \mathrm{a}$ & $15.5 \pm 1.73 \mathrm{~b}$ & $55 \pm 51.0 \mathrm{a}$ \\
$\mathrm{CM} 10$ & $16.70 \pm 2.57 \mathrm{ab}$ & $5.7 \pm 1.49 \mathrm{a}$ & $6.20 \pm 1.21 \mathrm{a}$ & $13.8 \pm 0.96 \mathrm{bc}$ & $0.697 \pm 0.037 \mathrm{a}$ & $9.51 \pm 1.68 \mathrm{a}$ & $13.3 \pm 1.84 \mathrm{ab}$ & $60 \pm 50.3 \mathrm{a}$ \\
$\mathrm{CM} 11$ & $17.20 \pm 2.66 \mathrm{ab}$ & $5.5 \pm 1.96 \mathrm{a}$ & $6.00 \pm 1.27 \mathrm{a}$ & $14.4 \pm 1.38 \mathrm{c}$ & $0.697 \pm 0.023 \mathrm{a}$ & $9.57 \pm 1.92 \mathrm{a}$ & $13.5 \pm 1.70 \mathrm{ab}$ & $60 \pm 50.3 \mathrm{a}$ \\
\hline $\mathrm{CM} 12$ & $15.70 \pm 2.26 \mathrm{a}$ & $3.9 \pm 0.99 \mathrm{a}$ & $5.20 \pm 1.11 \mathrm{a}$ & $12.1 \pm 0.83 \mathrm{a}$ & $0.685 \pm 0.068 \mathrm{a}$ & $10.19 \pm 2.28 \mathrm{a}$ & $15.6 \pm 5.62 \mathrm{~b}$ & $70 \pm 47.0 \mathrm{a}$ \\
\hline
\end{tabular}

Data are means \pm standard deviations. Means with different letters are significantly different at 0.05 level of probability. 
Effects of PVP, L-glutamine, myo-inositol and adenine on shoot elongation, rooting and plantlet acclimatization

After 3 months of culture on media containing PVP, Lglutamine, myo-inositol and/or adenine, shoot length, root number and length ranged from 12.05 to $14.98 \mathrm{~cm}, 3.2$ to 4.1 and 3.05 to $4.30 \mathrm{~cm}$, respectively (Table 4 ). As regards to the physiological characteristics, the chlorophyll content ranged from 15.2 to $31.8 \mathrm{CCI}$, the chlorophyll fluorescence ranged from 0.740 to 0.795 , the foliar surface ranged from 8.99 to $11.78 \mathrm{~cm}^{2}$ and the stomatal conductance ranged from 10.5 to $33.5 \mathrm{mmol} \mathrm{m}^{-2} \mathrm{~s}^{-1}$. The observed survival rates were between $60 \%$ and $85 \%$ and therefore did not exceed that obtained on the control medium (85\%). This result indicates that the addition of PVP, L-glutamine, myoinositol and adenine, alone or in combination to the elongation-rooting medium does not improve the survival rate during acclimatization as compared to CM1 medium. This shows that there is no need to use PVP, L-glutamine, myo-inositol and adenine at $1 \mathrm{~g} \mathrm{~L}^{-1}$ during shoot elongation and rooting.

Effects of carbon source on shoot elongation, rooting and plantlet acclimatization

In this experiment, there was no significant difference in terms of shoot length, root number and length when using different carbon sources. Shoot length ranged from 12.60 to $13.60 \mathrm{~cm}$, root number ranged from 3.1 to 3.6 and root length ranged from 2.95 to $3.85 \mathrm{~cm}$. Expectedly, it was found that sucrose affects the physiological characteristics of young plantlets and increases their survival rate during acclimatization. In fact, the shoots cultured on CM28 medium (i.e. containing sucrose) had thicker and larger leaves (Fig. 1) than those cultured on the other media. In addition, chlorophyll content foliar surface and stomatal conductance were significantly higher in shoots cultured on CM28 medium. This was reflected in a higher survival rate (90\%) during acclimatization (Fig. 2; Table 5).

The results of all these experiments suggest that CM28 medium, which was containing $6 \mathrm{~g} \mathrm{~L}^{-1}$ agar and $30 \mathrm{~g} \mathrm{~L}^{-1}$ sucrose, is the most effective for shoot elongation, rooting and plantlet acclimatization.

\section{Correlation between physiological characteristics and} plantlet acclimatization

As a final step of this study, the correlations between the measured physiological parameters and plantlet acclimatization using Pearson's correlation coefficients were assessed. Significant positive correlations were detected between chlorophyll content, chlorophyll fluorescence, foliar surface, stomatal conductance and plantlet survival during acclimatization (Table 6). This suggests that the physiological characteristics of young plantlets cultured in vitro affect their acclimatization to ex vitro conditions.

Table 4. Effect of PVP, adenine, L-glutamine and myo-inositol on shoot elongation, rooting, physiological characteristics and plantlet acclimatization

\begin{tabular}{|c|c|c|c|c|c|c|c|c|}
\hline Culture medium & $\begin{array}{c}\text { Shoot length } \\
(\mathrm{cm})\end{array}$ & $\begin{array}{c}\text { Number of roots } \\
\text { per shoot }\end{array}$ & Root length $(\mathrm{cm})$ & $\begin{array}{l}\text { Chlorophyll } \\
\text { content }(\mathrm{CCI})\end{array}$ & $\begin{array}{l}\text { Chlorophyll } \\
\text { fluorescence }\end{array}$ & $\begin{array}{c}\text { Foliar surface } \\
\left(\mathrm{cm}^{2}\right)\end{array}$ & $\begin{array}{l}\text { Stomatal conductance } \\
\left(\mathrm{mmol} \mathrm{m} \mathrm{m}^{-2} \mathrm{~s}^{-1}\right)\end{array}$ & $\begin{array}{c}\text { Plantlet } \\
\text { acclimatization }\end{array}$ \\
\hline CM13 & $14.07 \pm 3.07 \mathrm{a}$ & $3.9 \pm 1.79 a$ & $3.40 \pm 1.24 \mathrm{a}$ & $22.0 \pm 10.38 \mathrm{ab}$ & $0.788 \pm 0.011 \mathrm{fg}$ & $9.63 \pm 3.26 \mathrm{a}$ & $29.6 \pm 15.08 \mathrm{~cd}$ & $60 \pm 50.3 a$ \\
\hline CM14 & $14.24 \pm 5.26 \mathrm{a}$ & $3.3 \pm 1.34 \mathrm{a}$ & $3.60 \pm 1.90 \mathrm{a}$ & $17.4 \pm 9.22 \mathrm{ab}$ & $0.773 \pm 0.033 \mathrm{cdef}$ & $10.15 \pm 4.09 a$ & $19.4 \pm 7.00 \mathrm{abc}$ & $60 \pm 50.3 \mathrm{a}$ \\
\hline CM15 & $13.89 \pm 4.58 \mathrm{a}$ & $3.9 \pm 1.66 \mathrm{a}$ & $3.25 \pm 1.21 \mathrm{a}$ & $16.6 \pm 7.70 \mathrm{a}$ & $0.785 \pm 0.011 \mathrm{efg}$ & $11.1 \pm 4.06 \mathrm{a}$ & $22.4 \pm 12.43 \mathrm{bcd}$ & $65 \pm 48.9 a$ \\
\hline CM16 & $14.87 \pm 3.01 \mathrm{a}$ & $3.8 \pm 1.40 \mathrm{a}$ & $3.15 \pm 0.88 \mathrm{a}$ & $15.2 \pm 4.41 \mathrm{a}$ & $0.767 \pm 0.021 \mathrm{cde}$ & $10.76 \pm 3.94 \mathrm{a}$ & $15.0 \pm 5.52 \mathrm{ab}$ & $60 \pm 50.3 \mathrm{a}$ \\
\hline CM17 & $13.93 \pm 4.19 a$ & $3.5 \pm 0.97 \mathrm{a}$ & $3.30 \pm 1.09 \mathrm{a}$ & $24.6 \pm 7.71 \mathrm{ab}$ & $0.789 \pm 0.007 \mathrm{fg}$ & $11.78 \pm 2.14 \mathrm{a}$ & $23.6 \pm 6.10 \mathrm{bcd}$ & $80 \pm 41.0 \mathrm{a}$ \\
\hline CM18 & $12.60 \pm 3.10 \mathrm{a}$ & $3.8 \pm 1.55 \mathrm{a}$ & $3.05 \pm 1.64 \mathrm{a}$ & $17.5 \pm 0.97 \mathrm{ab}$ & $0.774 \pm 0.006 \mathrm{cdef}$ & $9.83 \pm 2.76 \mathrm{a}$ & $26.1 \pm 6.29 \mathrm{bcd}$ & $75 \pm 44.4 \mathrm{a}$ \\
\hline CM19 & $14.35 \pm 3.25 \mathrm{a}$ & $3.5 \pm 1.35 \mathrm{a}$ & $3.85 \pm 1.67 a$ & $19.3 \pm 12.70 \mathrm{ab}$ & $0.749 \pm 0.016 \mathrm{ab}$ & $9.90 \pm 2.02 \mathrm{a}$ & $22.1 \pm 5.82 \mathrm{bcd}$ & $75 \pm 44.4 \mathrm{a}$ \\
\hline CM20 & $12.05 \pm 3.31 \mathrm{a}$ & $3.3 \pm 1.06 \mathrm{a}$ & $3.95 \pm 1.88 \mathrm{a}$ & $16.7 \pm 1.07 \mathrm{a}$ & $0.759 \pm 0.007 \mathrm{bc}$ & $9.28 \pm 1.62 \mathrm{a}$ & $10.5 \pm 0.71 \mathrm{a}$ & $80 \pm 41.0 \mathrm{a}$ \\
\hline CM21 & $14.98 \pm 3.81 \mathrm{a}$ & $3.7 \pm 2.00 \mathrm{a}$ & $3.60 \pm 1.29 \mathrm{a}$ & $24.8 \pm 13.53 \mathrm{ab}$ & $0.779 \pm 0.007 \mathrm{defg}$ & $9.77 \pm 4.13 \mathrm{a}$ & $33.5 \pm 5.88 \mathrm{~d}$ & $80 \pm 41.0 \mathrm{a}$ \\
\hline $\mathrm{CM} 22$ & $13.52 \pm 2.12 \mathrm{a}$ & $3.2 \pm 1.03 \mathrm{a}$ & $3.80 \pm 1.65 \mathrm{a}$ & $18.9 \pm 14.52 \mathrm{ab}$ & $0.795 \pm 0.020 \mathrm{~g}$ & $11.77 \pm 1.35 \mathrm{a}$ & $32.6 \pm 18.57 \mathrm{~cd}$ & $75 \pm 44.4 \mathrm{a}$ \\
\hline CM23 & $14.35 \pm 2.50 a$ & $3.8 \pm 1.32 \mathrm{a}$ & $3.95 \pm 1.85 a$ & $16.4 \pm 1.30 \mathrm{a}$ & $0.760 \pm 0.006 \mathrm{bcd}$ & $10.27 \pm 4.06 \mathrm{a}$ & $29.8 \pm 11.07 \mathrm{~cd}$ & $75 \pm 44.4 \mathrm{a}$ \\
\hline $\mathrm{CM} 24$ & $14.92 \pm 4.44 \mathrm{a}$ & $4.1 \pm 2.23 \mathrm{a}$ & $3.30 \pm 1.09 \mathrm{a}$ & $20.0 \pm 10.70 \mathrm{ab}$ & $0.768 \pm 0.016 \mathrm{cde}$ & $8.99 \pm 2.13 \mathrm{a}$ & $30.1 \pm 7.30 \mathrm{~cd}$ & $70 \pm 47.0 \mathrm{a}$ \\
\hline $\mathrm{CM} 25$ & $13.94 \pm 2.82 \mathrm{a}$ & $3.6 \pm 1.58 \mathrm{a}$ & $4.30 \pm 2.00 \mathrm{a}$ & $28.3 \pm 4.72 \mathrm{ab}$ & $0.779 \pm 0.008$ defg & $10.31 \pm 3.43 \mathrm{a}$ & $31.9 \pm 9.86 \mathrm{~cd}$ & $85 \pm 36.6 a$ \\
\hline $\mathrm{CM} 26$ & $14.33 \pm 3.77 \mathrm{a}$ & $3.8 \pm 2.04 \mathrm{a}$ & $3.80 \pm 1.84 \mathrm{a}$ & $31.8 \pm 16.86 b$ & $0.780 \pm 0.011 \mathrm{efg}$ & $11.51 \pm 1.81 \mathrm{a}$ & $28.6 \pm 3.83 \mathrm{~cd}$ & $80 \pm 41.0 \mathrm{a}$ \\
\hline $\mathrm{CM} 27$ & $14.76 \pm 3.77 \mathrm{a}$ & $3.8 \pm 2.44 \mathrm{a}$ & $4.10 \pm 1.52 \mathrm{a}$ & $31.7 \pm 11.47 b$ & $0.740 \pm 0.008 \mathrm{a}$ & $9.14 \pm 1.53 \mathrm{a}$ & $29.4 \pm 1.76 \mathrm{~cd}$ & $75 \pm 44.4 \mathrm{a}$ \\
\hline
\end{tabular}

Data are means \pm standard deviations. Means with different letters are significantly different at 0.05 level of probability.

Table 5. Effect of carbon source on shoot elongation, rooting, physiological characteristics and plantlet acclimatization

\begin{tabular}{ccccccccc}
\hline $\begin{array}{c}\text { Culture } \\
\text { medium }\end{array}$ & $\begin{array}{c}\text { Shoot length } \\
(\mathrm{cm})\end{array}$ & $\begin{array}{c}\text { Number of } \\
\text { roots per } \\
\text { shoot }\end{array}$ & $\begin{array}{c}\text { Root length } \\
(\mathrm{cm})\end{array}$ & $\begin{array}{c}\text { Chlorophyll } \\
\text { content }(\mathrm{CCI})\end{array}$ & $\begin{array}{c}\text { Chlorophyll } \\
\text { fluorescence }\end{array}$ & $\begin{array}{c}\text { Foliar surface } \\
\left(\mathrm{cm}^{2}\right)\end{array}$ & $\begin{array}{c}\text { Stomatal } \\
\text { conductance } \\
\left(\mathrm{mmol} \mathrm{m}^{-2} \mathrm{~s}^{-1}\right)\end{array}$ & $\begin{array}{c}\text { Plantlet } \\
\text { acclimatization }\end{array}$ \\
\hline $\mathrm{CM} 28$ & $13.60 \pm 2.01 \mathrm{a}$ & $3.6 \pm 0.84 \mathrm{a}$ & $3.85 \pm 0.85 \mathrm{a}$ & $30.0 \pm 1.89 \mathrm{a}$ & $0.784 \pm 0.015 \mathrm{a}$ & $13.29 \pm 1.71 \mathrm{a}$ & $36.0 \pm 2.35 \mathrm{a}$ & $90 \pm 30.8 \mathrm{a}$ \\
$\mathrm{CM} 29$ & $12.80 \pm 1.62 \mathrm{a}$ & $3.2 \pm 0.79 \mathrm{a}$ & $2.95 \pm 1.28 \mathrm{a}$ & $28.0 \pm 1.18 \mathrm{~b}$ & $0.774 \pm 0.026 \mathrm{a}$ & $11.77 \pm 0.03 \mathrm{~b}$ & $33.3 \pm 3.86 \mathrm{a}$ & $70 \pm 47.0 \mathrm{a}$ \\
$\mathrm{CM} 30$ & $12.60 \pm 1.51 \mathrm{a}$ & $3.1 \pm 1.20 \mathrm{a}$ & $3.00 \pm 1.15 \mathrm{a}$ & $27.2 \pm 0.57 \mathrm{~b}$ & $0.770 \pm 0.022 \mathrm{a}$ & $11.29 \pm 0.01 \mathrm{~b}$ & $28.9 \pm 5.21 \mathrm{~b}$ & $75 \pm 44.4 \mathrm{a}$ \\
\hline
\end{tabular}

Table 6. Pearson's correlation coefficients between physiological characteristics and plantlet acclimatization

\begin{tabular}{ccccc}
\hline Variables & Chlorophyll content & Chlorophyll fluorescence & Foliar surface & Stomatal conductance \\
\hline Chlorophyll content & 1 & & & \\
Chlorophyll fluorescence &, $627^{* *}$ & 1 & 1 &, $564^{*}$ \\
Foliar surface &, $598^{* *}$ &, $568^{*}$ &, $652^{*}$ &, $640^{*}$ \\
Stomatal conductance &, $837^{* *}$ &, $696^{*}$ &, $637^{*}$ & 1 \\
Plantlet acclimatization &, $716^{*}$ & &
\end{tabular}


82

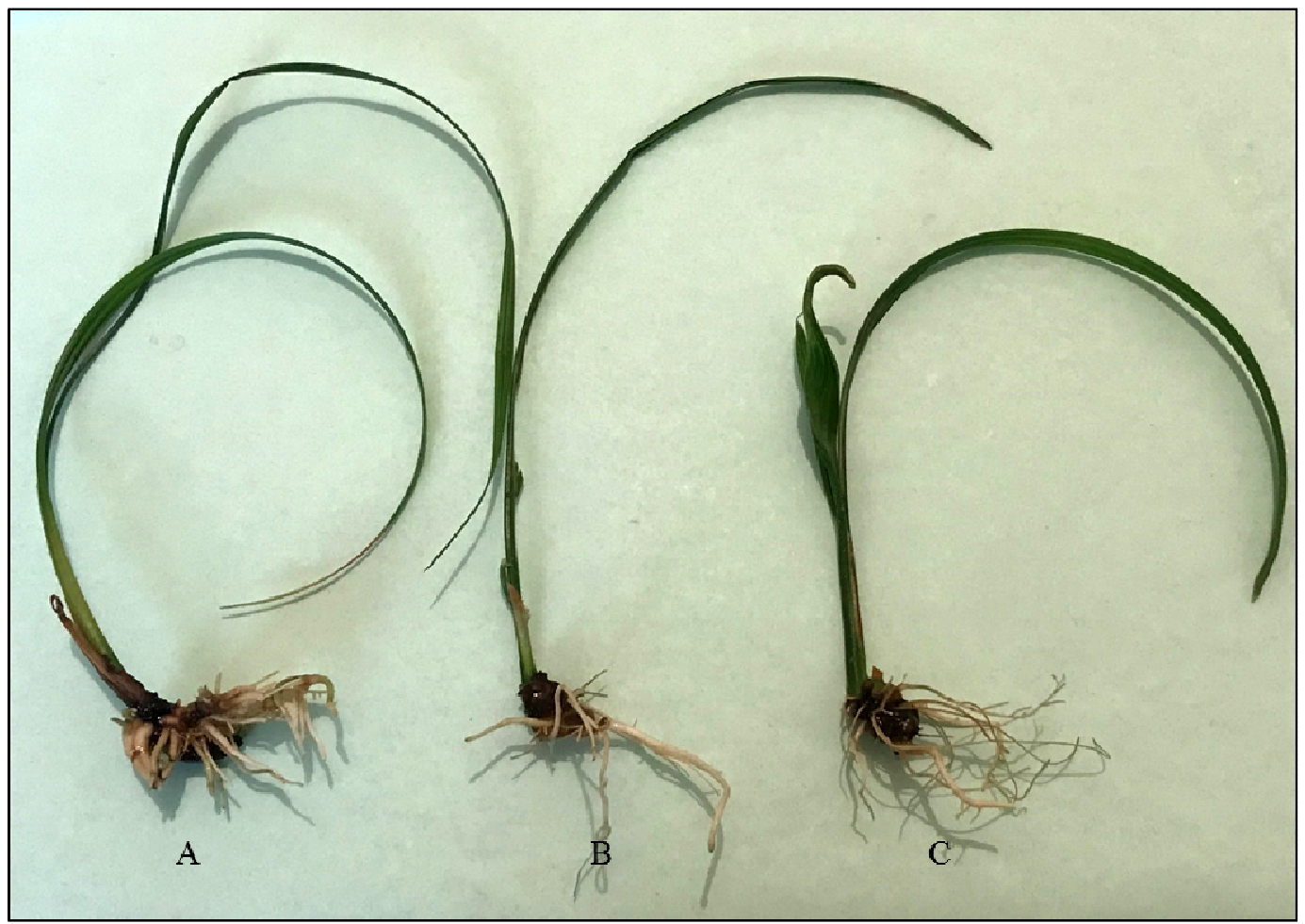

Fig. 1. Shoot elongation and rooting after 3 months of culture on : A MS/2 medium supplemented with $1 \mathrm{mg} \mathrm{L}^{-1} \mathrm{IBA}_{1} 1 \mathrm{mg} \mathrm{L} \mathrm{L}^{-1}$ NAA, $1 \mathrm{mg} \mathrm{L}^{-1}$ BAP, $1 \mathrm{mg} \mathrm{L}^{-1}$ kinetin, $30 \mathrm{~g} \mathrm{~L}^{-1}$ commercial granulated sugar and $6 \mathrm{~g} \mathrm{~L}^{-1}$ agar (CM11); B PGR-free liquid MS/2 medium supplemented with $30 \mathrm{~g} \mathrm{~L}^{-1}$ commercial granulated sugar (CM2); and C PGR-free MS/2 medium supplemented with 30 $\mathrm{g} \mathrm{L}^{-1}$ sucrose and $6 \mathrm{~g} \mathrm{~L}^{-1}$ agar (CM28).

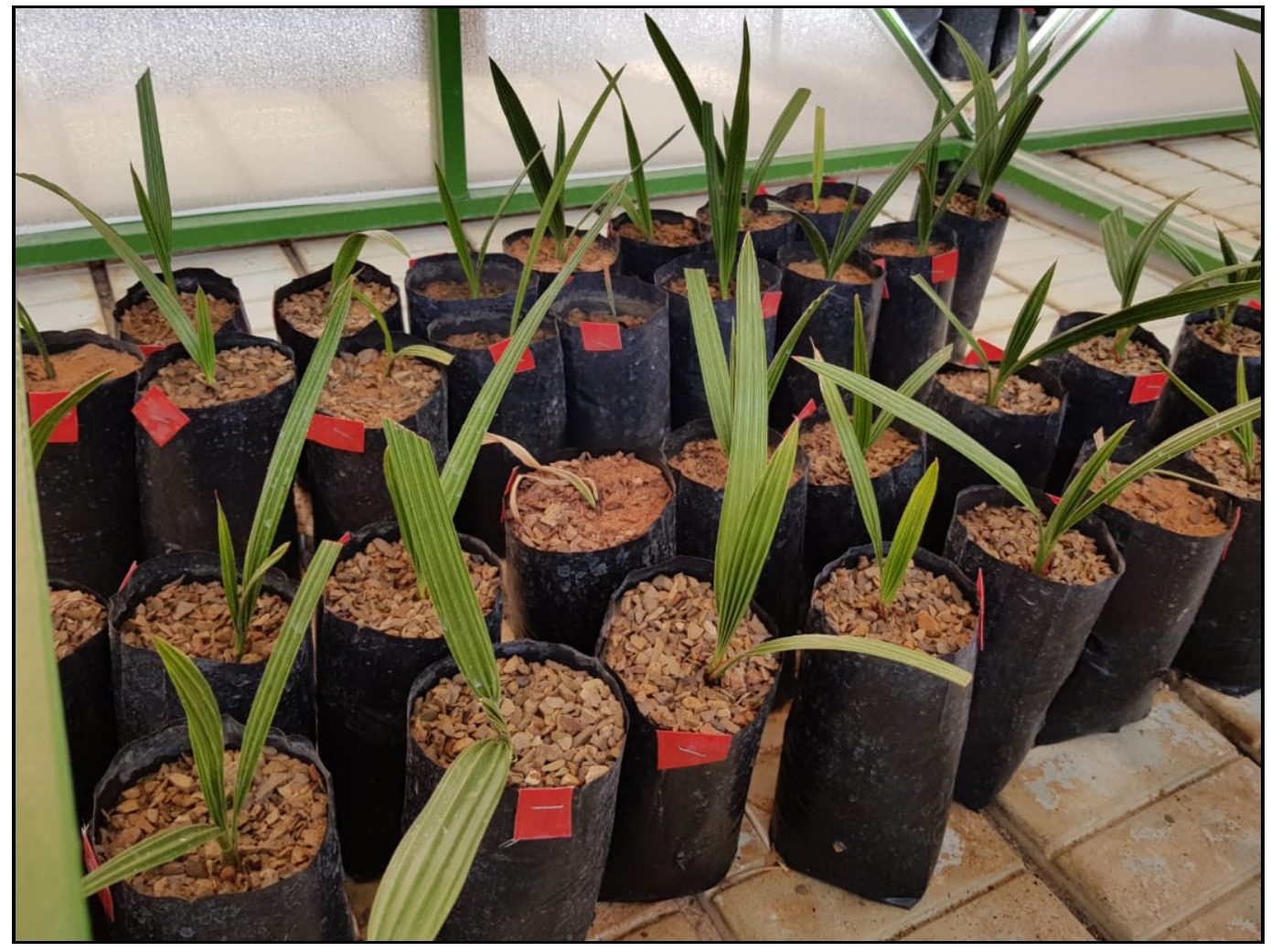

Fig. 2. Plantlet acclimatization: These plantlets were cultured on PGR-free MS/2 medium supplemented with $30 \mathrm{~g} \mathrm{~L}^{-1}$ sucrose and $6 \mathrm{~g} \mathrm{~L}^{-1}$ agar (CM28) during the elongation-rooting phase. 


\section{Discussion}

This study investigated the effects of several factors on shoot elongation and rooting and plantlet acclimatization of date palm cv. 'Mejhoul'. In many plant species, including date palm, it has been reported that liquid medium promotes shoot elongation in comparison with semi-solid medium (Skidmore et al., 1988; Suthar et al., 2011; Mazri, 2012). This is in good agreement with the results of the present study. Rezali et al. (2017) reported that liquid media facilitate nutrient uptake which stimulates plant growth. This might be the reason behind the longer shoots obtained in liquid medium. However, it appears that the shoots cultured on liquid medium are fragile and most of them fail to survive under the glasshouse conditions. In fact, the findings of the present study indicated that semi-solid medium resulted in higher plantlet quality (in terms of leaf thickness, width and greening) and a significantly higher acclimatization rate as compared to the liquid one. This supports previous findings on date palm cv. 'Boufeggous' (Mazri, 2015). In date palm and banana micropropagation, it has been suggested to transfer shoots from liquid to a semi-solid medium for few weeks before being transferred to the glasshouse in order to increase their survival rate (Bhagyalakshmi and Singh, 1995; Mazri, 2012).

The results of the present study support the general statement that plant growth regulators promote in vitro shoot elongation and rooting. Auxins and cytokinins are the most important classes of PGRs (Machakova et al., 2008). Accordingly, in many plant species, combinations of auxins and cytokinins were successfully used to stimulate shoot elongation and root induction. Auxins are known to control cell division and cell elongation and to promote adventitious root formation (Machakova et al., 2008). Cytokinins are essential for plant cell division and are very effective in promoting shoot bud formation and proliferation (Van Staden et al., 2008). GA 3 is a PGR that plays various roles. It may promote or inhibit shoot and root formation depending on the species, and has been successfully used for shoot elongation (Moshkov et al., 2008). Our results indicated that $\mathrm{GA}_{3}$ increases shoot and root lengths in comparison with PGR-free semi-solid medium (CM1). This is in good agreement with the findings of Khierallah and Bader (2007) who demonstrated that increasing $\mathrm{GA}_{3}$ concentration increased shoot length in date palm cv. 'Maktoom'.

In the present investigation, PGRs resulted in longer shoots with higher number of roots compared to PGR-free semi-solid medium. However, the shoots cultured on PGRfree semi-solid medium showed greener, thicker and more vigorous leaves. This is in good agreement with previous findings on date palm cv. 'Najda' (Mazri and Meziani, 2013). Interestingly, it has been found that shoots that were cultured on CM1 medium exhibit higher survival rate during acclimatization than those cultured on PGRcontaining media. Accordingly, the use of PGR-free medium for shoot elongation and rooting of date palm cv. 'Mejhoul' is recommended.
L-Glutamine, myo-inositol, adenine and PVP are widely used in plant tissue culture. L-Glutamine is an amino acid that promotes and maintains cell function (Newsholme et al., 2003). In date palm micropropagation, L-glutamine was used to promote adventitious bud and somatic embryo proliferation (Zouine et al., 2005; Zouine and El Hadrami, 2007; Mazri et al., 2016). Myo-inositol is a vitamin that promotes cell division (Kiviharju et al., 2005). Adenine, also known as vitamin B4, has been used in plant tissue culture for its effects similar to those of cytokinins (Thorpe et al., 2008; Van Staden et al., 2008), while PVP is a widely used compound in date palm organogenesis to control tissue browning. In previous studies on cv. 'Mejhoul' organogenesis, L-glutamine and myo-inositol showed their beneficial effect during shoot bud multiplication (Mazri et al., 2016) whereas PVP has been successfully used as an antibrowning agent during adventitious bud initiation and shoot bud multiplication (Meziani et al., 2016). Herein, the effects of these compounds as well as adenine were evaluated on shoot elongation, rooting and plantlet acclimatization. However, none of them has improved the survival rate after transfer to ex vitro conditions. Accordingly, the use of a medium without these compounds is suggested. This will contribute to cost saving during the organogenesis process.

Carbon sources play both nutritional and osmotic roles in plant micropropagation, and supply the necessary energy for plant cell and tissue growth and morphogenesis (Neto and Otoni, 2003). Sucrose is by far the most used carbon source in plant micropropagation (Yaseen et al., 2013). The data presented here show that shoot quality and plantlet survival after ex vitro acclimatization were improved by the addition of sucrose to elongation and rooting medium. This is in good agreement with previous results on date palm cv. '16-bis' (Mazri, 2014). Sucrose has been used for the micropropagation of many date palm cultivars through either somatic embryogenesis or organogenesis (e.g. Fki et al., 2011; Mazri et al., 2016; Mazri et al., 2017). This may be due to its beneficial effect on adventitious bud and somatic embryo induction and proliferation.

Chlorophyll content and fluorescence, foliar surface and stomatal conductance are physiological characteristics that determine plant quality. To the best of our knowledge, this is the first study that measures these physiological parameters in date palm cv. 'Mejhoul' plantlets obtained in vitro. Our results indicated that the composition of the culture medium affects the physiological characteristics of date palm plantlets. This is in good agreement with results from the literature. In fact, in many other plant species, it has been found that medium composition and culture conditions affect the physiological characteristics of plants (e.g. Lu, 2005; Mohamed and Alsadon, 2010). In the present investigation, chlorophyll content and fluorescence, foliar surface and stomatal conductance showed a positive correlation with plantlet survival during acclimatization. This suggests that high values of these physiological parameters are reflected in high plantlet quality and high survival rate under ex vitro conditions. Accordingly, they can be considered as good indicators for successful transplantation. 
84

\section{Conclusions}

Date palm organogenesis has gained recently much interest because of its high multiplication potential and production of true to type plantlets. This research demonstrated how culture conditions during the elongation-rooting phase affect shoot growth and their physiological characteristics as well as plantlet acclimatization. It has been found that some medium additives such as L-glutamine, myo-inositol, adenine, PVP and PGRs are not needed to achieve high levels of acclimatization. This is very important in developing a costeffective regeneration system for date palm. Regarding carbon sources, sucrose has improved plantlet quality and increased the survival rate during acclimatization. However, it is important to carry out an economic analysis to determine whether its beneficial effect justifies its high cost.

\section{Acknowledgements}

This work was supported by the MESRSFC and CNRST (Morocco), project number PPR2/2016/36.

\section{References}

Bhagyalakshmi, Singh NS (1995). Role of liquid versus agar-gelled media in mass propagation and ex vitro survival in bananas. Plant Cell Tissue and Organ Culture 41(1):71-73.

Burrows FJ, Milthorpe FL (1976). Stomatal conductance in the control of gas exchange. In: Kozlowski TT (Ed) Water deficits and plant growth, vol.IV.Academic Press, London New York, pp 103-152.

Cabrera HM, Argandoña VH, Zúñiga GE, Corcuera LJ (1995). Effect of infestation by aphids on the water status of barley and insect development. Phytochemistry 40(4):1083-1088.

Camen D, Beinșan C,Șumălan R, Pasc M (2010). Research on the effect of arbuscular mycorrhizae on some physiological indices of lettuce-Lactuca sativa L. Journal of Horticulture, Forestry and Biotechnology 14(3):128-130.

Derridj S, Wu BR, Stammitti L, Garrec JP, Derrien A (1996). Chemicals on the leaf surface, information about the plant available to insects. Entomologia Experimentalis et Applicata 80(1):197-201.

Filella I, Penuelas J, Seco R (2009). Short-chained oxygenated VOC emissions in Pinus halepensis in response to changes in water availability. Acta Physiologiae Plantarum 31(2):311-318.

Fki L, Bouaziz N, Kriaa W, Benjemaa-Masmoudi R, Gargouri-Bouzid R, Rival A, Drira N (2011). Multiple bud cultures of 'Barhee' date palm (Phoenix dactylifera) and physiological status of regenerated plants. Journal of Plant Physiology 168(14):16941700.

Jain SM (2012). Date palm biotechnology: Current status and prospectivean overview. Emirates Journal of Food and Agriculture 24(5):386-399.

Johnson DV (2011). Introduction: Date palm biotechnology from theory to practice. In: Jain SM, Al-Khayri JM, Johnson DV (Eds). Date palm biotechnology. Springer, Dordrecht pp 1-11.

Johnson HB, Rowlands PG, TingIP (1979). Tritium and carbon-14double isotope porometer for simultaneous measurements of transpiration and photosynthesis. Photosynthetica 13(4):409-418.
Jones HG (1992). Plants and microclimate: a quantitative approach to environmental plant physiology. Cambridge University Press.

Khan S, Bi Bi T (2012). Direct shoot regeneration system for date palm (Phoenix dactylifera L.) cv. 'Dhakki' as a means of micropropagation. Pakistan Journal ofBotany 44(6):1965-1971.

Khierallah HSM, Bader SM (2007). Micropropagation of date palm (Phoenix dactylifera L.) var. 'Mektoom' through direct organogenesis. Acta Horticulturae 736:213-224.

Kiviharju E, Moisander S, Laurila J (2005). Improved green plant regeneration rates from oat anther culture and the agronomic performance of some DH lines. Plant Cell, Tissue and Organ Culture 81(1):1-9.

Lee B, Kim D, Ryu CM (2008). A super-absorbent polymer combination promotes bacterial aggressiveness uncoupled from the epiphytic population. The Plant PathologyJournal 24(3):283-288.

Lu MC (2005). Micropropagation of Vitis thunbergii Sieb. et Zucc,, a medicinal herb, through high-frequency shoot tip culture. Scientia Horticulturae 107(1):6469.

Machakova I, Zazimalova E, George EF (2008). Plant growth regulators I: Introduction; auxins, their analogues and inhibitors. In: George EF, Hall MA, De Klerk GJ (Eds). Plant propagation by tissue culture, 3rd edn. Springer, Dordrecht pp 175-204.

Mazri MA (2012). Effect of liquid media and in vitro pre-acclimatization stage on shoot elongation and acclimatization of date palm (Phoenix dactylifera L.) cv. 'Najda'. Journal of Ornamental and Horticultural Plants 2(4):225-231.

Mazri MA (2013). Effect of basal medium, explants size and density on the in vitro proliferation and growth of date palm (Phoenix dactylifera $\mathrm{L}$.) cultivar' '16-bis'. Notulae Scientia Biologicae 5(3):332-337.

Mazri MA (2014). Effects of plant growth regulators and carbon source on shoot proliferation and regeneration in date palm (Phoenix dactylifera L.) '16-bis'. Journal of Horticultural Science and Biotechnology 89(4):415422.

Mazri MA (2015). Role of cytokinins and physical state of the culture medium to improve in vitro shoot multiplication, rooting and acclimatization of date palm (Phoenix dactylifera L.) cv. 'Boufeggous'. Journal of Plant Biochemistry and Biotechnology 24(3):268-275.

Mazri MA, Meziani R(2013). An improved method for micropropagation and regeneration of date palm (Phoenix dactylifera L.). Journal of Plant Biochemistry and Biotechnology 22(2):176-184.

Mazri MA, Meziani R (2015). Micropropagation of date palm: a review. Cell \&Developmental Biology 4(3):160.

Mazri MA, Meziani R, El Fadile J, Ezzinbi A (2016). Optimization of medium composition for in vitro shoot proliferation and growth of date palmcv.'Mejhoul'.3 Biotech 6(1):111.

Mazri MA, Belkoura I, Meziani R, Mokhless B, Nour S (2017). Somatic embryogenesis from bud and leaf explants of date palm (Phoenix dactylifera L.) cv.'Najda'3Biotech 7(1):58.

Meziani R, Jaiti F, Mazri MA, Anjarne M, Ait Chitt M, El Fadile J, Alem C (2015). Effects of plant growth regulators and light intensity on the micropropagation of date palm (Phoenix dactylifera L.) cv. 'Mejhoul'. Journal ofCrop Science and Biotechnology 18(5):325-331. 
Meziani R, Jaiti F, Mazri MA, Hassani A, Ben Salem S, ... Alem C (2016). Organogenesis of Phoenix dactylifera L. cv. 'Mejhoul': Influences of natural and synthetic compounds on tissue browning, and analysis of protein concentrations and peroxidase activity in explants. Scientia Horticulturae 204:145-152.

Mohamed MAH, Alsadon AA (2010). Influence of ventilation and sucrose on growth and leaf anatomy of micropropagated potato plantlets. ScientiaHorticulturae 123(3):295-300.

Moshkov IE, Novikova GV, Hall MA, George EF (2008). Plant growth regulators III: gibberellins, ethylene, abscisic acid, their analogues and inhibitors; miscellaneous compounds. In: George EF, Hall MA, De Klerk GJ (Eds). Plant propagation by tissue culture, 3rd edn. Springer, Dordrecht pp 227-281.

Murashige T, Skoog FA (1962). A revised medium for rapid growth and bioassays with tobacco tissue cultures. Phys Planta Physiologia Plantarum 15(3):473-479.

Neto VBP, Otoni WC (2003). Carbon sources and their osmotic potential in plant tissue culture: does it matter? Scientia Horticulturae 97(34):193-202.

Newsholme P,Lima MMR, Procopio J, Pithon-Curi TC, ... Curi R(2003). Glutamine and glutamate as vital metabolites. Brazilian Journal of Medical and Biological Research 36(2):153-163.

Oliveira RS, Ma Y, Rocha I, Carvalho MF, Vosátka M, Freitas H (2016). Arbuscular mycorrhizal fungi are an alternative to the application of chemical fertilizer in the production of the medicinal and aromatic plant Coriandrum sativum L. Journal of Toxicology and Environmental Health A79(7):320-328.

RezaliNI, SidikNJ,Saleh A, Osman NI, AdamNAM(2017). The effects of different strength of MS media in solid and liquid media on in vitro growth of Typhonium flagelliforme. Asian Pacific Journal of Tropical Biomedicine 7(2):151-156.

Sedra MH (2005). Phenological descriptors and molecular markers for the determination of true-to-type of tissue culture-derived plants using organogenesis of some Moroccan date palm (Phoenix dactylifera L.) varieties. Al Awamia 113:85-101.

Sedra MH (2011). Development of new Moroccan selected date palm varieties resistant to Bayoud and of good fruit quality. In: Jain SM, AlKhayri JM, Johnson DV (Eds). Date palm biotechnology. Springer, Dordrecht pp 513-531.
Sedra MH (2015). Date palm status and perspective in Morocco. In: AlKhayri JM, Jain SM, Johnson DV (Eds). Date Palm Genetic Resources and Utilization. Springer,Dordrecht pp 257-323.

Shabani F, Kumar L, Taylor S (2014). Projecting date palm distribution in Iran under climate change using topography, physicochemical soil properties, soil taxonomy, land use and climate data. Theoretical and Applied Climatology 118(3):553-567.

Skidmore DJ, Simons AJ, Bedi S (1988).In vitro culture of shoots of Pinus caribaea on a liquid medium. Plant Cell Tissue and Organ Culture 14(2):129-136.

Suthar RK, Habibi N, Purohit SD (2011). Influence of agar concentration and liquid medium on in vitro propagation ofBoswellia serrata Roxb. Indian Journal of Biotechnology 10:224227.

Thorpe T, Stasolla C, Yeung EC, de Klerk GJ, Roberts A, George EF (2008). The components of plant tissue culture media II: organic additions, osmotic and $\mathrm{pH}$ effects, and support systems. In: George EF, Hall MA, De Klerk GJ (Eds). Plant propagation by tissue culture, 3rd edn. Springer, Dordrecht pp 115-173.

Van Staden J, Zazimalova E, George EF (2008). Plant growth regulators II: cytokinins, their analogues and antagonists. In: George EF, Hall MA, De Klerk GJ (Eds). Plant propagation by tissue culture, 3rd edn. Springer, Dordrecht pp 205-226.

Vernon LP, Seely GR (1966). The chlorophylls. Academic Press, New York. Yaseen M, Ahmad T, Sablok G, Standardi A, Hafiz IA (2013). Review: role of carbon sources for in vitro plant growth and development. Molecular Biology Reports 40:2834-2849.

Zaid A, El-Korchi B, Visser HJ (2011). Commercial date palm tissue culture procedures and facility establishment. In: Jain SM, Al-Khayri JM, Johnson DV (Eds). Date palm biotechnology. Springer, Dordrecht pp 137-180.

Zouine J, El Hadrami I (2007). Effect of 2,4D, glutamine and BAP on embryogenic suspension culture of date palm (Phoenix dactylifera L). ScientiaHorticulturae 112(2):221-226.

Zouine J, El Bellaj M, Meddich A, Verdeil J, El Hadrami I (2005). Proliferation and germination of somatic embryos from embryogenic suspension cultures in Phoenix dactylifera L. Plant Cell Tissue and Organ Culture 82(1):83-92. 